\title{
Latest Results from the Daya Bay Experiment
}

\section{Tadeáš Dohnal $^{a, *}$ on behalf of the Daya Bay Collaboration}

(a complete list of authors can be found at the end of the proceedings)

${ }^{a}$ Institute of Particle and Nuclear Physics, Faculty of Mathematics and Physics, Charles University V Holešovičkách 2, 18000 Prague 8, Czech Republic

E-mail: dohnal@ipnp.mff.cuni.cz

The Daya Bay Reactor Neutrino Experiment, located in southeast China, was designed to measure short baseline oscillation of electron antineutrinos originating from six $2.9 \mathrm{GW}_{\text {th }}$ nuclear reactors. Since 2011, it has collected an unprecedented sample of millions of reactor antineutrino candidates, the largest sample in the world up to date, which led to the discovery of the non-zero $\theta_{13}$ mixing angle just in 2012. In this talk, we present an overview of the latest results from Daya Bay including the measurement of oscillation parameters driving the reactor antineutrino disappearance at short baseline, with the most precise measurement of the $\theta_{13}$ mixing angle in the world, search for light sterile neutrino mixing, and search for electron antineutrinos associated with gravitational wave events among others.

$37^{\text {th }}$ International Cosmic Ray Conference (ICRC 2021)

July 12th - 23rd, 2021

Online - Berlin, Germany

\footnotetext{
${ }^{*}$ Presenter
} 


\section{The Daya Bay Reactor Neutrino Experiment}

The Daya Bay Reactor Neutrino Experiment was designed to measure the $\theta_{13}$ mixing angle by studying reactor antineutrino disappearance at a $\sim 2 \mathrm{~km}$ baseline due to neutrino oscillation. It began data taking in late 2011 and finished operation at the end of 2020. The location of the experiment is in proximity of Daya Bay and Ling Ao nuclear power plants, about $55 \mathrm{~km}$ northeast from Hong Kong in south China. The power plants contain in total six $2.9 \mathrm{GW}_{\text {th }}$ pressurized water reactors making them one of the most powerful nuclear complexes in the world. Each reactor isotropically emits $\sim 6 \times 10^{20} \bar{v}_{e}$ /s originating in beta-decays of fission fragments, which makes them an intense and pure source of $\bar{v}_{e}$ 's with energies up to $\sim 10 \mathrm{MeV}$.

The Daya Bay experiment nominally operated with 8 antineutrino detectors (ADs) located in 3 experimental halls (EHs), as shown in the left panel of Fig. 1. The near experimental halls (EH1 \& EH2, each containing 2 ADs) are located $400 \mathrm{~m}$ from nuclear cores with the aim to sample the reactor $\bar{v}_{e}$ flux and spectrum only minimally affected by neutrino oscillation. The distance between the far hall (EH3 containing 4 ADs) and the nuclear cores is $\sim 1700 \mathrm{~m}$, where the maximal disappearance of reactor $\bar{v}_{e}$ due to neutrino oscillation driven by the $\theta_{13}$ mixing angle can be observed. All EHs are placed underground in order to suppress cosmic rays and associated backgrounds, with EH3 being the deepest one.
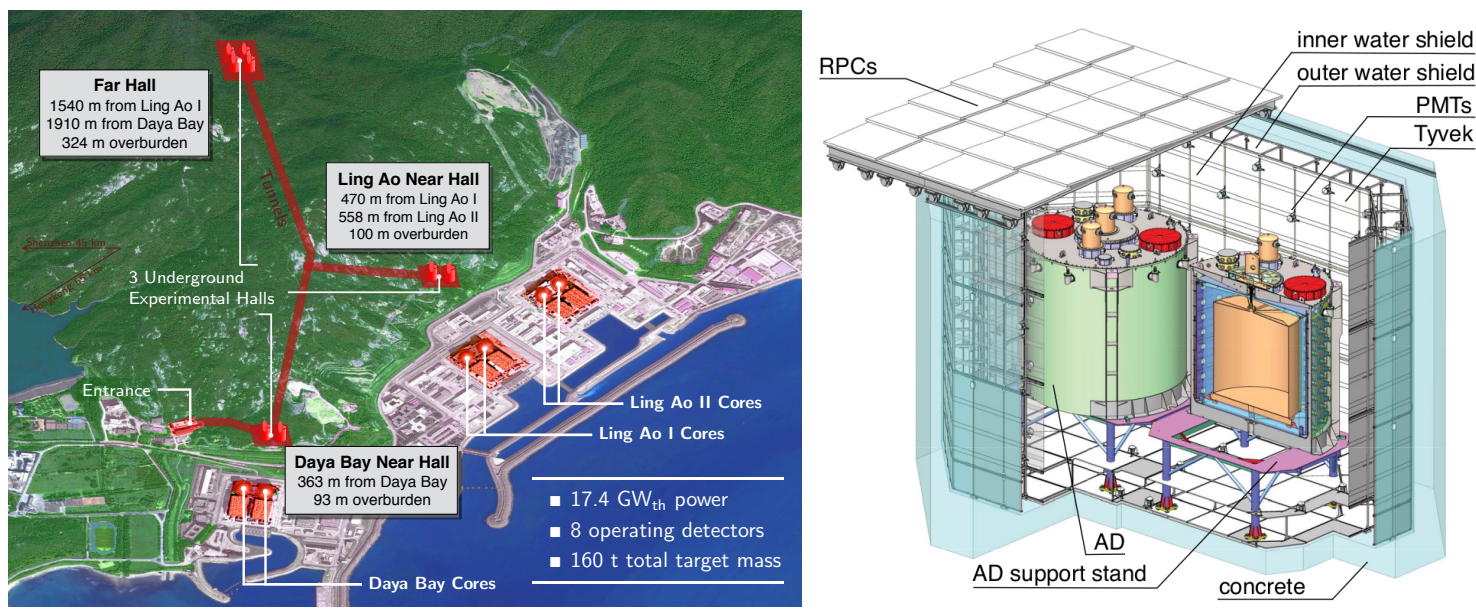

Figure 1: (Left) Layout of the Daya Bay experiment. Six reactor cores serve as a powerful and pure source of $\bar{v}_{e}$ 's. Mostly unoscillated flux and spectrum is sampled by two near experimental halls (EH1 \& EH2) while the far hall (EH3) sees the largest effect of the oscillation driven by $\theta_{13}$ mixing angle. (Right) Section of the detectors in one of the near halls. The ADs are submerged in an instrumented pool of ultra pure water covered with RPCs. Segmentation into three volumes is shown in the section of the AD to the right.

Each AD consists of 3 nested cylindrical regions. The innermost contains $20 \mathrm{t}$ of liquid scintillator doped with gadolinium (GdLS) and serves as the main target. It is surrounded by $22 \mathrm{t}$ of liquid scintillator without doping (LS) which detects gammas that escape GdLS (and also serves as a target). These two volumes are then nested in non-scintillating mineral oil working as a buffer. There are 192 inward-facing 8-inch photomultiplier tubes collecting scintillation light mounted around the perimeter of the mineral oil volume. 
The ADs are submerged in an instrumented water pool which serves both as passive shielding against cosmogenic neutrons and ambient radioactivity and as an active Cherenkov detectors for cosmic-ray muons. The water pool is covered by an array of 4-layer Resistive Plate Chambers (RPCs) for further improvement of muon detection.

Reactor antineutrinos are detected via the inverse beta decay (IBD) reaction: $\bar{v}_{e}+p \rightarrow e^{+}+n$. As liquid scintillator is a hydrocarbon compound, free protons are abundant there for antineutrino to interact with. Positron created in IBD quickly looses energy and eventually annihilates emitting two gammas, which also promptly deposit their energy. Neutron, on the other hand, thermalizes first and then is captured on a nucleus. In GdLS, it is most likely gadolinium (nGd) with mean capture time $\sim 30 \mu \mathrm{s}$. Gamma cascade is emitted with energy $\sim 8 \mathrm{MeV}$. In LS, neutrons are mainly captured on hydrogen $(\mathrm{nH})$ with mean capture time $\sim 200 \mu \mathrm{s}$. A single $2.2 \mathrm{MeV}$ gamma is then emitted. The spatial $(\lesssim 50 \mathrm{~cm})$ and temporal coincidence of the prompt positron signal and the delayed neutron capture allows for a powerful background rejection in general. The energy of the prompt signal can be directly related to the incoming antineutrino energy as $E_{\bar{v}_{e}} \simeq E_{\text {prompt }}+0.78 \mathrm{MeV}$.

\section{Neutrino Oscillation Results}

The survival probability of $\bar{v}_{e}$ for short baseline experiment such as Daya Bay is given approximately (in framework of 3 active neutrino flavors)

$$
P\left(\bar{v}_{e} \rightarrow \bar{v}_{e}\right) \simeq 1-\sin ^{2}\left(2 \theta_{13}\right) \sin ^{2}\left(\frac{\Delta m_{e e}^{2} L}{4 E}\right),
$$

where $\Delta m_{e e}^{2}$ is an effective mass splitting $\left(\sin ^{2} \frac{\Delta m_{e e}^{2} L}{4 E} \simeq \cos ^{2} \theta_{12} \sin ^{2} \frac{\Delta m_{31}^{2} L}{4 E}+\sin ^{2} \theta_{12} \frac{\Delta m_{32}^{2} L}{4 E}\right), L$ is the distance traversed by neutrino and $E$ is its energy.

Daya Bay was the first experiment to discover the non-zero value of the $\theta_{13}$ mixing angle just in 2012 using nGd sample. Since then, several improved measurements have been published with the latest one corresponding to 1958 days of data taking [1]. During that time, unprecedented sample of over 3.9 million IBD candidates with almost 0.5 million in the far hall (EH3) had been collected.

By comparing flux and spectrum shape across all ADs (the difference between the near halls and the far hall being essential) as shown in the left panel of Fig. 2 the values of the parameters driving the oscillation can be determined with the results $\sin ^{2} 2 \theta_{13}=0.0856 \pm 0.0029$ and $\Delta m_{e e}^{2}=$ $\left(2.522_{-0.070}^{+0.068}\right) \times 10^{-3} \mathrm{eV}^{2}$. The best fit and the confidence regions are shown in the right panel of Fig. 2. Statistics contributed about $60 \%$ to the total uncertainty of $\sin ^{2} 2 \theta_{13}$ and $50 \%$ for $\Delta m_{e e}^{2}$. Daya Bay's measurement of $\sin ^{2} 2 \theta_{13}$ is the world's most precise one and it is expected to be further improved to $<3 \%$ precision with the final data set. The $\Delta m_{32}^{2}$ mass squared difference is also measured with a leading precision comparable to that achieved by accelerator experiments.

The neutrino oscillation measurement can be also performed using IBD sample with neutron capture on hydrogen $(\mathrm{nH})$. So far, analysis comparing antineutrino rates across all ADs based on 621 days of data taking has been performed leading to the result $\sin ^{2} 2 \theta_{13}=0.071 \pm 0.011$ [2]. Measurement based on $\mathrm{nH}$ is largely independent from the $\mathrm{nGd}$ measurement, since the statistical samples are completely different and systematical uncertainties are mostly decoupled. Analysis using $\mathrm{nH}$ suffers from larger systematics, especially larger background dominated by the accidental 

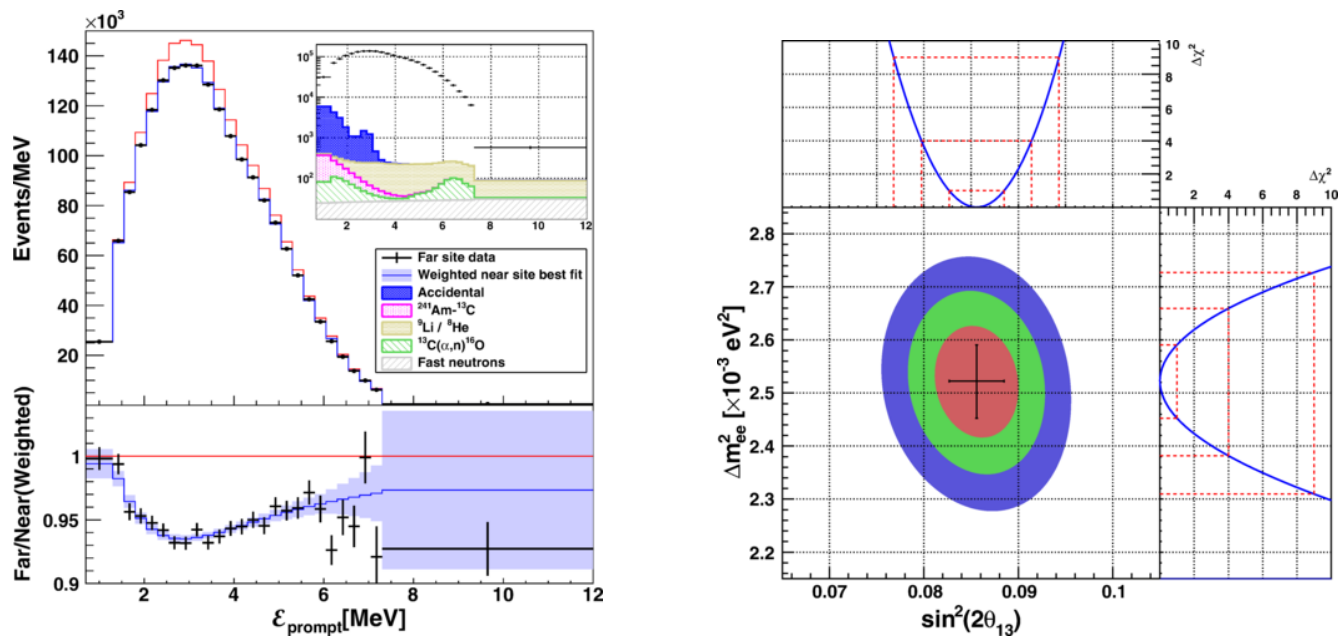

Figure 2: (Left) Energy spectrum of reactor antineutrinos detected in the far hall ADs (black points) is compared to the prediction based on the near halls data assuming no oscillations (red line) and with the best oscillation fit (blue line). (Right) The best fit of $\sin ^{2} 2 \theta_{13}$ and $\Delta m_{e e}^{2}$ and the 1,2,3 $\sigma$ confidence regions.

coincidence of two uncorrelated signals. A spectral analysis is under preparation, likely to become one of the leading measurements of $\sin ^{2} 2 \theta_{13}$ in the world.

\section{Search for Sterile Neutrino Mixing}

Despite the success of the three-neutrino mixing model, several experimental anomalies can be explained by the existence of sterile neutrino(s). Daya Bay searched for light sterile neutrino mixing within the minimal extension 3 (active) +1 (sterile) neutrino model. With its unique layout with multiple baselines, the experiment can explore several orders of $\left|\Delta m_{41}^{2}\right|$. The existence of an extra neutrino could appear in the data as an additional spectrum distortion driven by the new mass-squared difference $\Delta m_{41}^{2}$. However, no deviation from the three-neutrino model was found analyzing a data set acquired over 1230 days [3]. Thus Daya Bay was able to place limits on sterile neutrino mixing $\sin ^{2} 2 \theta_{14}$ for three orders of magnitude in $\left|\Delta m_{41}^{2}\right|$, yielding the most stringent constrains up to date for $\left|\Delta m_{41}^{2}\right|<0.2 \mathrm{eV}^{2}$, as shown in the left panel of Fig. 3.

This measurement was extended by the limits from Bugey-3 [7] and further combined with the results from the MINOS and MINOS+ experiments [8] to scrutinize the allowed sterile neutrino parameter space of the LSND [9] and MiniBooNE [10] experiments. The excess of electron (anti)neutrinos in the muon (anti)neutrino beam might be explained by sterile neutrino mixing with $\Delta m_{41}^{2} \sim 1 \mathrm{eV}^{2}$ and an effective mixing angle $\sin ^{2} 2 \theta_{\mu e} \equiv \sin ^{2} 2 \theta_{14} \sin ^{2} \theta_{24}$. The $\theta_{14}$ and $\theta_{24}$ mixing angles can be directly constrained by the electron and muon (anti)neutrino disappearance experiments respectively, hence the combination of Daya Bay and Bugey-3 with MINOS and MINOS+. Since no deviation from a three-neutrino paradigm was observed, the combination yielded strong constrains on the sterile neutrino mixing over seven orders of magnitude in $\Delta m_{41}^{2}$. The LSND and MiniBooNE 99\% C.L. allowed regions were excluded at $99 \% \mathrm{CL}_{s}$ for $\Delta m_{41}^{2}<1.2 \mathrm{eV}^{2}$, as shown in right panel of Fig. 3, suggesting that excess in those experiments might not be caused 

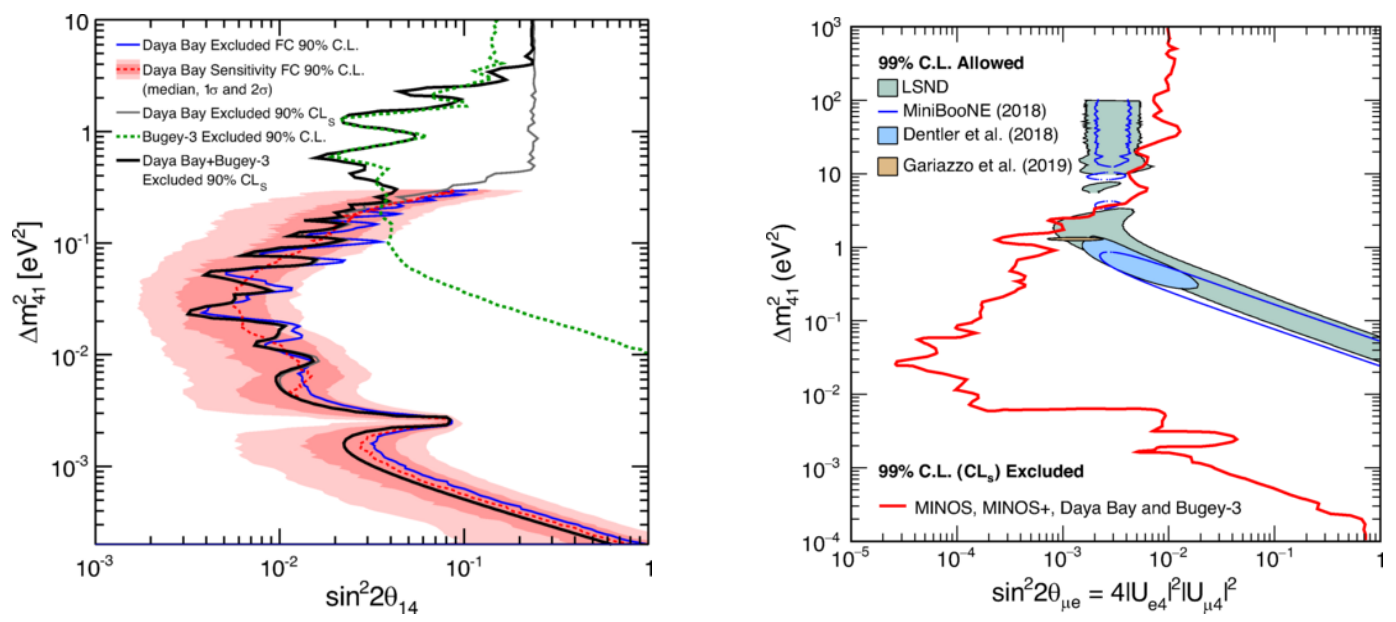

Figure 3: (Left) Exclusion region at 90\% C.L. from Daya Bay obtained with the Feldman-Cousins (red) and $\mathrm{CL}_{s}$ (gray) statistical methods, and its extension with the Bugey-3 data (black). (Right) Exclusion region at $99 \% \mathrm{CL}_{s}$ from the combination of the Daya Bay, Bugey-3, MINOS and MINOS+ experiments, together with the 99\% C.L. allowed region from the LSND and MiniBooNE experiments, is shown. A global fit $[4,5]$ as well as a fit to appearance experiments only [6] is ruled out at $>99 \%$ C.L. The regions to the right of the curves are excluded.

by sterile neutrinos. Furthermore, global fit to the experiments searching for sterile neutrinos $[4,5]$ as well as fit only to the appearance experiments [6] were fully excluded on more than 99\% C.L..

\section{Measurement of Reactor Antineutrino Flux and Spectrum}

Precise measurement of reactor $\bar{v}_{e}$ flux was performed using 2.2 million IBD candidates collected in the near halls over 1230 days [11]. The average IBD yield was determined to be $(5.91 \pm 0.09) \times 10^{-43} \mathrm{~cm}^{2} /$ fission. While it is consistent with previous experimental results as shown in the left panel of Fig. 4, when compared to Huber-Mueller model $[12,13]$ the ratio of measured to predicted is $0.952 \pm 0.014 \pm 0.023$, where the first uncertainty is experimental and the second one from the theoretical model. This discrepancy is called 'reactor antineutrino anomaly'.

Using the same data set, correlations between reactor core fuel evolution and changes in the reactor antineutrino flux and energy spectrum were also analyzed [14]. Fuel-dependent variation in the IBD yield was observed (as shown in the right panel of Fig. 4) rejecting the the hypothesis of a constant antineutrino flux as a function of the ${ }^{239} \mathrm{Pu}$ fission fraction at 10 standard deviations and the hypothesis of a constant antineutrino energy spectrum at 5.1 standard deviations. Individual yields of the two most prominent fissile isotopes ${ }^{235} \mathrm{U}$ and ${ }^{239} \mathrm{Pu}$ were determined to be $(6.17 \pm 0.17)$ and $(4.27 \pm 0.26) \times 10^{-43} \mathrm{~cm}^{2} /$ fission respectively (see the left panel of Fig. 5) suggesting that ${ }^{235} \mathrm{U}$ is the primary contributor to the reactor antineutrino anomaly.

Along with improved measurement of the prompt energy spectrum of reactor antineutrinos, individual antineutrino spectra from ${ }^{235} \mathrm{U}$ and ${ }^{239} \mathrm{Pu}$ fission were extracted using evolution of the prompt spectrum as a function of the isotope fission fractions based on 3.5 million IBD candidates collected in the near halls over 1958 days [15]. Total spectrum showed discrepancy of over 5 

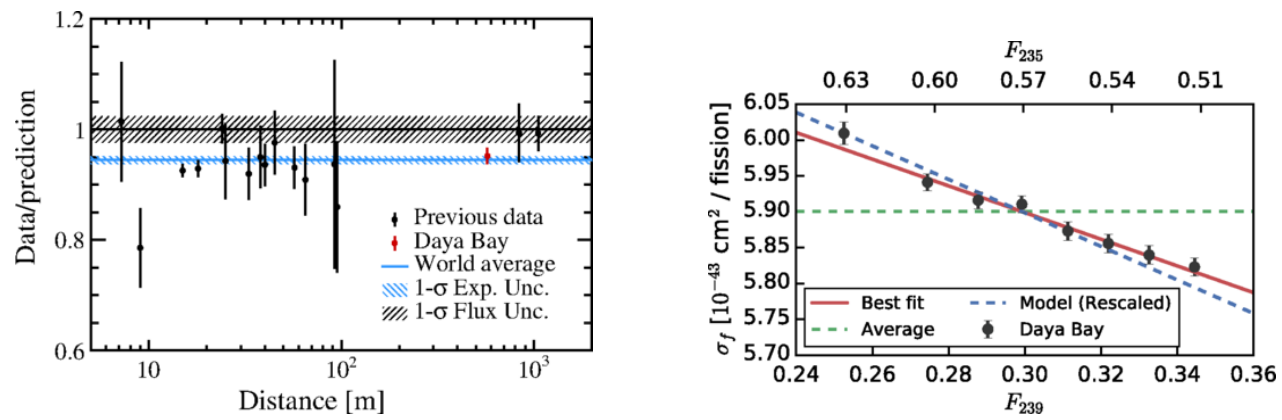

Figure 4: (Left) The ratio of measured reactor antineutrino yield to the Huber-Mueller theoretical prediction as a function of the distance from the reactor to detector. Each ratio is corrected for the effect of neutrino oscillation. The Daya Bay measurement is shown at the flux weighted baseline $(578 \mathrm{~m})$ of the two near halls. (Right) IBD yield per fission, $\sigma_{f}$, versus effective ${ }^{239} \mathrm{Pu}$ (lower axis) or ${ }^{235} \mathrm{U}$ (upper axis) fission fraction.

standard deviations when compared to Huber-Mueller model, especially significant in 4-6 MeV 'bump' region ( $>6$ standard deviations).
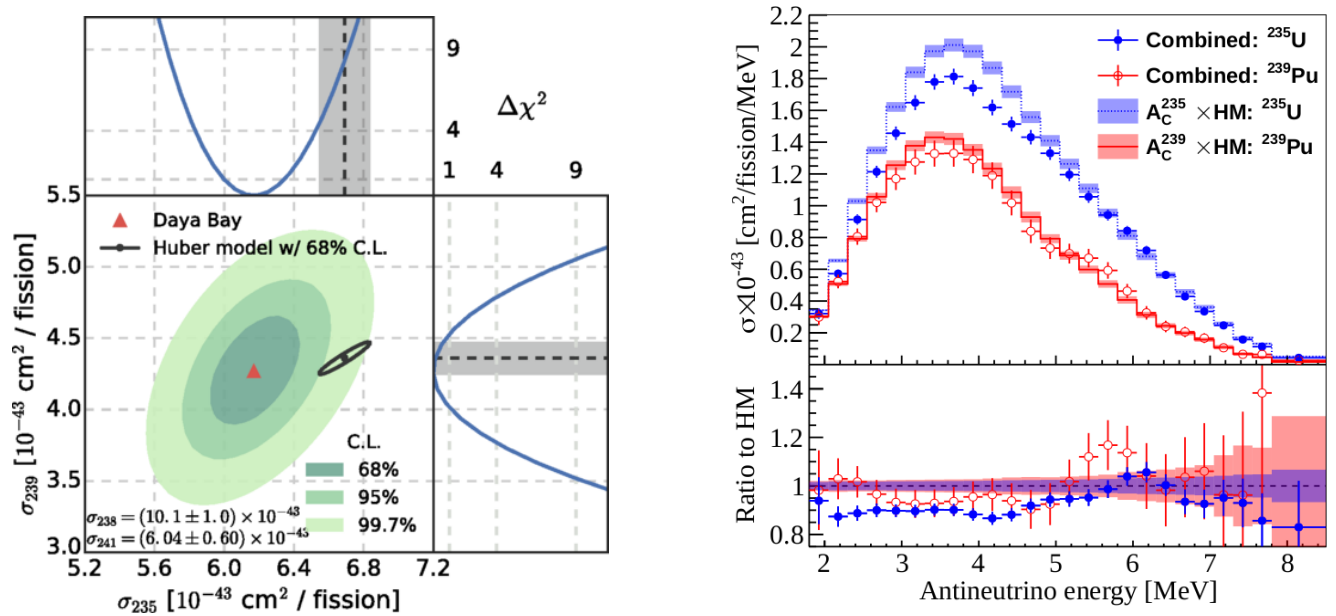

Figure 5: (Left) Combined measurement of ${ }^{235} \mathrm{U}$ and ${ }^{239} \mathrm{Pu}$ IBD yields per fission $\sigma_{235}$ and $\sigma_{239}$. The red triangle indicates the best fit with corresponding contours in green while prediction of Huber-Mueller model is shown in black. (Right) Top $-{ }^{235} \mathrm{U}$ and ${ }^{239} \mathrm{Pu}$ antineutrino spectra unfolded from the jointly deconvolved Daya Bay and PROSPECT measurements. Bottom - ratio of the measurements to their respective models.

Joint analysis with the PROSPECT experiment, which detects $\bar{v}_{e}$ 's from highly enriched uranium compact research reactor core, was also performed [16]. It showed that the measured ${ }^{235} \mathrm{U}$ antineutrino spectra are consistent with each other. Combined analysis then reduced correlation between ${ }^{235} \mathrm{U}$ and ${ }^{239} \mathrm{Pu}$ spectra (in the right panel of Fig. 5) and ${ }^{235} \mathrm{U}$ spectrum shape uncertainty.

\section{Search for $\bar{v}_{\mathrm{e}}$ 's Associated with Gravitational Waves}

The establishment of a possible connection between neutrino emission and gravitational-wave (GW) bursts is important to our understanding of the physical processes that occur when black holes 
or neutron stars merge. Search for $\bar{v}_{e}$ that coincided with observation of GWs was performed using data spanning from 2011 to August 2017 a thus covering GW150914, GW151012, GW151226, GW170104, GW170608, GW170814, and GW170817 [17]. Three time windows of $\pm 10, \pm 500$, and $\pm 1000 \mathrm{~s}$ relative to the occurrence of the GW events were studied. Correlated neutrino candidates were searched in energy range of 1.8 to $100 \mathrm{MeV}$. The detected $\bar{v}_{e}$ candidates were consistent with the expected background rates for all the three time windows. Thus upper limits (90\% confidence level) of the $\bar{v}_{e}$ fluence were determined assuming monochromatic spectra and are shown in Fig. 6. If Fermi-Dirac spectrum is assumed, the upper limits of the $\bar{v}_{e}$ fluence were found to be $(5.4-7.0) \times 10^{9} \mathrm{~cm}^{-2}$ for the three time windows.

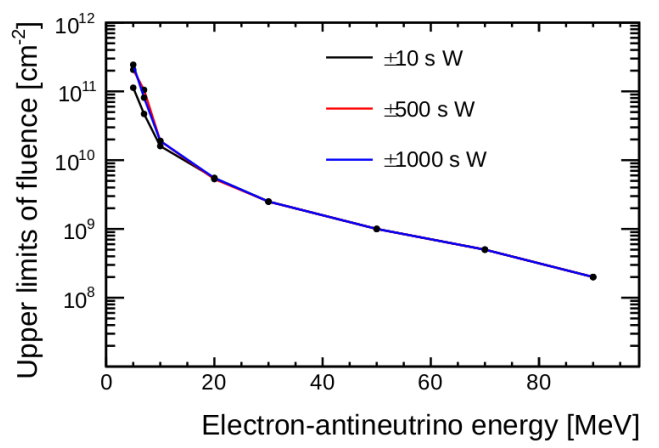

Figure 6: Upper limit (90\% C.L.) of the $\bar{v}_{e}$ fluence for each energy point for the GW-coincident event search assuming monochromatic spectra. Three curves are shown for the three time windows (W).

\section{Summary}

The recent measurement of reactor antineutrino oscillation at the Daya Bay experiment provides the most precise determination of the $\theta_{13}$ mixing angle and an estimate of $\left|\Delta m_{32}^{2}\right|$ with competitive precision to accelerator experiments. An improved search for light sterile neutrino mixing results in the world's most stringent limits for $\left|\Delta m_{41}^{2}\right|<0.2 \mathrm{eV}^{2}$. The combination of Daya Bay and Bugey-3 with MINOS and MINOS+ ruled out a significant part of allowed sterile neutrino parameter space of the LSND and MiniBooNE experiments. Reactor $\bar{v}_{e}$ flux was measured with the result consistent with previous experimental results but below the prediction of the Huber-Mueller model. The total reactor $\bar{v}_{e}$ spectrum was also obtained, again showing discrepancy with the Huber-Mueller model. By using fuel evolution, individual $\bar{v}_{e}$ yields and spectra for ${ }^{235} \mathrm{U}$ and ${ }^{239} \mathrm{Pu}$ were also extracted. No excess $\bar{v}_{e}$ 's associated with gravitational wave events were observed.

\section{References}

[1] DAYA BAY collaboration, Measurement of the electron antineutrino oscillation with 1958 days of operation at Daya Bay, Phys. Rev. Lett. 121 (2018) 241805.

[2] DAYA BAy collaboration, New measurement of $\theta_{13}$ via neutron capture on hydrogen at Daya Bay, Phys. Rev. D 93 (2016) 072011. 
[3] DAYA BAY AND MINOS + collaboration, Improved constraints on sterile neutrino mixing from disappearance searches in the MINOS, MINOS+, Daya Bay, and Bugey-3 experiments, Phys. Rev. Lett. 125 (2020) 071801.

[4] S. Gariazzo, C. Giunti, M. Laveder and Y.F. Li, Updated global 3+1 analysis of short-baseline neutrino oscillations, Journal of High Energy Physics 6 (2017) 135.

[5] S. Gariazzo, C. Giunti, M. Laveder and Y. Li, Model-independent $\bar{v}_{e}$ short-baseline oscillations from reactor spectral ratios, Physics Letters B 782 (2018) 13.

[6] M. Dentler, Álvaro Hernández-Cabezudo, J. Kopp, P. Machado, M. Maltoni, I. Martinez-Soler et al., Updated global analysis of neutrino oscillations in the presence of eV-scale sterile neutrinos, Journal of High Energy Physics 8 (2018) 10.

[7] B. Achkar, R. Aleksan, M. Avenier, G. Bagieu, J. Bouchez, R. Brissot et al., Search for neutrino oscillations at 15, 40 and 95 meters from a nuclear power reactor at Bugey, Nuclear Physics B 434 (1995) 503.

[8] MINOS+ collaboration, Search for sterile neutrinos in MINOS and MINOS+ using a two-detector fit, Phys. Rev. Lett. 122 (2019) 091803.

[9] LSND collaboration, Evidence for neutrino oscillations from the observation of $\bar{v}_{e}$ appearance in a $\bar{v}_{\mu}$ beam, Phys. Rev. D 64 (2001) 112007.

[10] MiniBooNE collaboration, Significant excess of electronlike events in the MiniBooNE short-baseline neutrino experiment, Phys. Rev. Lett. 121 (2018) 221801.

[11] DAYA BAY collaboration, Improved measurement of the reactor antineutrino flux at Daya Bay, Phys. Rev. D 100 (2019) 052004.

[12] P. Huber, Determination of antineutrino spectra from nuclear reactors, Phys. Rev. C 84 (2011) 024617.

[13] T.A. Mueller, D. Lhuillier, M. Fallot, A. Letourneau, S. Cormon, M. Fechner et al., Improved predictions of reactor antineutrino spectra, Phys. Rev. C 83 (2011) 054615.

[14] DaYA BAy collaboration, Evolution of the reactor antineutrino flux and spectrum at Daya Bay, Phys. Rev. Lett. 118 (2017) 251801.

[15] DAYA BAY collaboration, Extraction of the ${ }^{235} \mathrm{U}$ and ${ }^{239} \mathrm{Pu}$ antineutrino spectra at Daya Bay, Phys. Rev. Lett. 123 (2019) 111801.

[16] F.P. An, M. Andriamirado, A.B. Balantekin, H.R. Band, C.D. Bass, D.E. Bergeron et al., Joint determination of reactor antineutrino spectra from ${ }^{235} U$ and ${ }^{239} P$ fission by Daya Bay and PROSPECT, 2021.

[17] DAYA BAY collaboration, Search for electron-antineutrinos associated with gravitational-wave events GW150914, GW151012, GW151226, GW170104, GW170608, GW170814, and GW170817 at Daya Bay, Chinese Physics C 45 (2021) 055001. 


\section{Full Authors List: Daya Bay Collaboration}

F. P. $\mathrm{An}^{1}$, A. B. Balantekin ${ }^{2}$, M. Bishai ${ }^{3}$, S. Blyth ${ }^{4}$, G. F. $\mathrm{Cao}^{5}$, J. Cao ${ }^{5}$, J. F. Chang ${ }^{5}$, Y. Chang ${ }^{6}$, H. S. Chen ${ }^{5}$, S. M. Chen ${ }^{7}$, Y. Chen ${ }^{8,9}$, Y. X. Chen ${ }^{10}$, J. Cheng ${ }^{5}$, Z. K. Cheng ${ }^{9}$, J. J. Cherwinka ${ }^{2}$, M. C. Chu ${ }^{11}$, J. P. Cummings ${ }^{12}$, O. Dalager ${ }^{13}$, F. S. Deng ${ }^{14}$, Y. Y. Ding ${ }^{5}$, M. V. Diwan ${ }^{3}$, T. Dohnal ${ }^{15}$, D. Dolzhikov ${ }^{16}$, J. Dove ${ }^{17}$, M. Dvořák ${ }^{15}$, D. A. Dwyer ${ }^{18}$, J. P. Gallo ${ }^{19}$, M. Gonchar ${ }^{16}$, G. H. Gong 7 , H. Gong ${ }^{7}$, M. Grassi ${ }^{5,13}$, W. Q. Gu ${ }^{3}$, J. Y. Guo ${ }^{9}$, L. Guo ${ }^{7}$, X. H. Guo ${ }^{20}$, Y. H. Guo ${ }^{21}$, Z. Guo ${ }^{7}$, R. W. Hackenburg ${ }^{3}$,

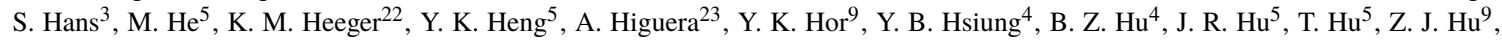
H. X. Huang ${ }^{24}$, J. H. Huang ${ }^{5}$, X. T. Huang ${ }^{25}$, Y. B. Huang ${ }^{26}$, P. Huber ${ }^{27}$, D. E. Jaffe ${ }^{3}$, K. L. Jen ${ }^{28}$, X. L. Ji ${ }^{5}$, X. P. Ji ${ }^{3}$, R. A. Johnson ${ }^{29}$, D. Jones ${ }^{30}$, L. Kang ${ }^{31}$, S. H. Kettell ${ }^{3}$, S. Kohn ${ }^{32}$, M. Kramer ${ }^{18,32}$, T. J. Langford ${ }^{22}$, J. Lee ${ }^{18}$, J. H. C. Lee ${ }^{33}$, R. T. Lei ${ }^{31}$, R. Leitner ${ }^{15}$, J. K. C. Leung ${ }^{33}$, F. $\mathrm{Li}^{5}$, H. L. $\mathrm{Li}^{5}$, J. J. $\mathrm{Li}^{7}$, Q. J. $\mathrm{Li}^{5}$, R. H. Li ${ }^{5}$, S. $\mathrm{Li}^{31}$, S. C. $\mathrm{Li}^{27}$, W. D. $\mathrm{Li}^{5}, \mathrm{X} . \mathrm{N} . \mathrm{Li}^{5}$, X. Q. $\mathrm{Li}^{34}, \mathrm{Y}^{2} \mathrm{~F}_{\mathrm{Li}} \mathrm{Li}^{5}$, Z. B. Li ${ }^{9}$, H. Liang ${ }^{14}$, C. J. Lin ${ }^{18}$, G. L. $\operatorname{Lin}^{28}$, S. Lin ${ }^{31}$, J. J. Ling 9 , J. M. Link ${ }^{27}$, L. Littenberg ${ }^{3}$, B. R. Littlejohn ${ }^{19}$, J. C. Liu ${ }^{5}$, J. L. $\mathrm{Liu}^{35}$, J. X. $\mathrm{Liu}^{5}$, C. Lu ${ }^{36}$, H. Q. $\mathrm{Lu}^{5}$, J. S. $\mathrm{Lu}^{5}$, K. B. $\mathrm{Luk}^{32,18}$, B. Z. Ma ${ }^{25}$, X. B. $\mathrm{Ma}^{10}$, X. Y. Ma ${ }^{5}$, Y. Q. $\mathrm{Ma}^{5}$, R. C. Mandujano ${ }^{13}$, C. Marshall ${ }^{18}$, K. T. McDonald ${ }^{36}$, R. D. McKeown ${ }^{37,38}$, Y. Meng ${ }^{35}$, J. Napolitano ${ }^{30}$, D. Naumov ${ }^{16}$, E. Naumova ${ }^{16}$, T. M. T. Nguyen ${ }^{28}$, J. P. Ochoa-Ricoux ${ }^{13}$, A. Olshevskiy ${ }^{16}$, H.-R. Pan ${ }^{4}$, J. Park ${ }^{27}$, S. Patton ${ }^{18}$, J. C. Peng ${ }^{17}$, C. S. J. Pun ${ }^{33}$, F. Z. Qi ${ }^{5}$, M. Qi ${ }^{39}$, X. Qian ${ }^{3}$, N. Raper ${ }^{9}$, J. Ren ${ }^{24}$, C. Morales Reveco ${ }^{13}$, R. Rosero ${ }^{3}$, B. Roskovec ${ }^{13}$, X. C. Ruan ${ }^{24}$, H. Steiner ${ }^{32,18}$, J. L. Sun ${ }^{40}$, T. Tmej ${ }^{15}$, K. Treskov ${ }^{16}$, W.-H. Tse ${ }^{11}$, C. E. Tull ${ }^{18}$, B. Viren ${ }^{3}$, V. Vorobel ${ }^{15}$, C. H. Wang ${ }^{6}$, J. Wang ${ }^{9}$, M. Wang ${ }^{25}$, N. Y. Wang ${ }^{20}$, R. G. Wang ${ }^{5}$, W. Wang ${ }^{9,38}$, W. Wang ${ }^{39}$, X. Wang ${ }^{41}$, Y. Wang ${ }^{39}$, Y. F. Wang ${ }^{5}$, Z. Wang ${ }^{5}$, Z. Wang ${ }^{7}$, Z. M. Wang ${ }^{5}$, H. Y. Wei ${ }^{3}$, L. H. Wei ${ }^{5}$, L. J. Wen ${ }^{5}$, K. Whisnant ${ }^{42}$, C. G. White ${ }^{19}$, H. L. H. Wong ${ }^{32,18}$, E. Worcester ${ }^{3}$, D. R. Wu ${ }^{5}$, F. L. Wu ${ }^{39}$, Q. Wu ${ }^{25}$, W. J. Wu ${ }^{5}$, D. M. Xia ${ }^{43}$, Z. Q. Xie ${ }^{5}$, Z. Z. Xing ${ }^{5}$, H. K. Xu ${ }^{5}$, J. L. Xu ${ }^{5}$, T. Xu ${ }^{7}$, T. Xue ${ }^{7}$, C. G. Yang ${ }^{5}$, L. Yang ${ }^{31}$, Y. Z. Yang ${ }^{7}$, H. F. Yao ${ }^{5}$, M. Ye ${ }^{5}$, M. Yeh ${ }^{3}$, B. L. Young ${ }^{42}$, H. Z. Yu ${ }^{9}$, Z. Y. Yu ${ }^{5}$, B. B. Yue ${ }^{9}$, V. Zavadskyi ${ }^{16}$, S. Zeng ${ }^{5}$, Y. Zeng ${ }^{9}$, L. Zhan ${ }^{5}$, C. Zhang ${ }^{3}$, F. Y. Zhang ${ }^{35}$, H. H. Zhang ${ }^{9}$, J. W. Zhang ${ }^{5}$, Q. M. Zhang ${ }^{21}$, S. Q. Zhang ${ }^{9}$, X. T. Zhang ${ }^{5}$, Y. M. Zhang ${ }^{9}$, Y. X. Zhang ${ }^{40}$, Y. Y. Zhang ${ }^{35}$, Z. J. Zhang ${ }^{31}$, Z. P. Zhang ${ }^{14}$, Z. Y. Zhang ${ }^{5}$, J. Zhao ${ }^{5}$, R. Z. Zhao ${ }^{5}$ L. Zhou ${ }^{5}$ H. L. Zhuang ${ }^{5}$ and J. H. Zou ${ }^{5}$

${ }^{1}$ Institute of Modern Physics, East China University of Science and Technology, Shanghai. ${ }^{2}$ University of Wisconsin, Madison, Wisconsin 53706. ${ }^{3}$ Brookhaven National Laboratory, Upton, New York 11973. ${ }^{4}$ Department of Physics, National Taiwan University, Taipei. ${ }^{5}$ Institute of High Energy Physics, Beijing. ${ }^{6}$ National United University, Miao-Li. ${ }^{7}$ Department of Engineering Physics, Tsinghua University, Beijing. ${ }^{8}$ Shenzhen University, Shenzhen. ${ }^{9}$ Sun Yat-Sen (Zhongshan) University, Guangzhou. ${ }^{10}$ North China Electric Power University, Beijing. ${ }^{11}$ Chinese University of Hong Kong, Hong Kong. ${ }^{12}$ Siena College, Loudonville, New York 12211. ${ }^{13}$ Department of Physics and Astronomy, University of California, Irvine, California 92697. ${ }^{14}$ University of Science and Technology of China, Hefei. ${ }^{15}$ Charles University, Faculty of Mathematics and Physics, Prague. ${ }^{16}$ Joint Institute for Nuclear Research, Dubna, Moscow Region. ${ }^{17}$ Department of Physics, University of Illinois at Urbana-Champaign, Urbana, Illinois $61801 .{ }^{18}$ Lawrence Berkeley National Laboratory, Berkeley, California 94720. ${ }^{19}$ Department of Physics, Illinois Institute of Technology, Chicago, Illinois 60616. ${ }^{20}$ Beijing Normal University, Beijing. ${ }^{21}$ Department of Nuclear Science and Technology, School of Energy and Power Engineering, Xi'an Jiaotong University, Xi'an. ${ }^{22}$ Wright Laboratory and Department of Physics, Yale University, New Haven, Connecticut 06520. ${ }^{23}$ Department of Physics, University of Houston, Houston, Texas 77204. ${ }^{24}$ China Institute of Atomic Energy, Beijing. ${ }^{25}$ Shandong University, Jinan. ${ }^{26}$ Guangxi University, No.100 Daxue East Road, Nanning. ${ }^{27}$ Center for Neutrino Physics, Virginia Tech, Blacksburg, Virginia 24061. ${ }^{28}$ Institute of Physics, National Chiao-Tung University, Hsinchu. ${ }^{29}$ Department of Physics, University of Cincinnati, Cincinnati, Ohio 45221. ${ }^{30}$ Department of Physics, College of Science and Technology, Temple University, Philadelphia, Pennsylvania 19122. ${ }^{31}$ Dongguan University of Technology, Dongguan. ${ }^{32}$ Department of Physics, University of California, Berkeley, California 94720. ${ }^{33}$ Department of Physics, The University of Hong Kong, Pokfulam, Hong Kong. ${ }^{34}$ School of Physics, Nankai University, Tianjin. ${ }^{35}$ Department of Physics and Astronomy, Shanghai Jiao Tong University, Shanghai Laboratory for Particle Physics and Cosmology, Shanghai. ${ }^{36}$ Joseph Henry Laboratories, Princeton University, Princeton, New Jersey $08544 .{ }^{37}$ California Institute of Technology, Pasadena, California 91125. ${ }^{38}$ College of William and Mary, Williamsburg, Virginia 23187. ${ }^{39}$ Nanjing University, Nanjing. ${ }^{40}$ China General Nuclear Power Group, Shenzhen. ${ }^{41}$ College of Electronic Science and Engineering, National University of Defense Technology, Changsha. ${ }^{42}$ Iowa State University, Ames, Iowa 50011. ${ }^{43}$ Chongqing University, Chongqing. 